\title{
Acute effects of different inspiratory resistive loading on heart rate variability in healthy elderly patients
}

\author{
Bruno Archiza ${ }^{1}$, Rodrigo P. Simões ${ }^{1}$, Renata G. Mendes ${ }^{1}$, \\ Guilherme A. F. Fregonezi ${ }^{2}$, Aparecida M. Catai ${ }^{1}$, Audrey Borghi-Silva ${ }^{1}$
}

\begin{abstract}
Background: The cardiovascular system is noticeably affected by respiration. However, whether different inspiratory resistive loading intensities can influence autonomic heart rate (HR) modulation remains unclear. Objective: The objective was to investigate HR modulation at three different inspiratory resistive loading intensities in healthy elderly men. Method: This was a prospective, randomized, double-blind study that evaluated 25 healthy elderly men. Cardiac autonomic modulation was assessed using heart rate variability (HRV) indices. All of the volunteers underwent maximal inspiratory pressure (MIP) measurements according to standardized pulmonary function measurements. Three randomly-applied inspiratory resistive loading (30, 60 and 80\% of MIP) intensities were then applied using an inspiratory resistance device (POWERbreathe, Southam, UK), during which the volunteers were asked to inhale for 2 seconds and exhale for 3 seconds and complete 12 breaths per minute. Each effort level was performed for 4 minutes, and HR and the distance between 2 subsequent $\mathrm{R}$ waves of electrocardiogram (R-R intervals) were collected at rest and at each intensity for further HRV analysis. Results: The parasympathetic HRV (rMSSD, SD1 and HF) indices demonstrated lower values at $80 \%$ (rMSSD: $19 \pm 2 \mathrm{~ms}, \mathrm{SD} 1: 13 \pm 2 \mathrm{~ms}$ and HF: $228 \pm 61 \mathrm{~ms}^{2}$ ) than at $30 \%$ MIP (rMSSD: $25 \pm 3 \mathrm{~ms}$, SD1: $18 \pm 2 \mathrm{~ms}$ and HF: $\left.447 \pm 95 \mathrm{~ms}^{2} ; \mathrm{p}<0.05\right)$. Conclusions: Lower inspiratory resistive loading intensities promoted a marked and positive improvement of parasympathetic sinus node modulation.
\end{abstract}

Keywords: physical therapy; respiratory muscle strength; maximal respiratory pressure; cardiac autonomic function; elderly.

\section{HOW TO CITE THIS ARTICLE}

Archiza B, Simões RP, Mendes RG, Fregonezi GAF, Catai AM, Borghi-Silva A. Acute effects of different inspiratory resistive loading on heart rate variability in healthy elderly patients. Braz J Phys Ther. 2013 July-Aug; 17(4):401-408. http://dx.doi.org/10.1590/ S1413-35552012005000100

\section{Introduction}

Cardiopulmonary physical therapy intervention involves techniques to help restore pulmonary volume, respiratory muscle strength (RMS), functional capacity and ameliorate post-surgical complications ${ }^{1,2}$. In this context, respiratory muscle training (RMT) is a commonly used resource that fosters improvement in both respiratory and peripheral muscle strength and provides functional capacity benefits in the elderly as well as in chronic cardiopulmonary disease patients ${ }^{3,4}$.

The aging process and some chronic diseases can reduce RMS ${ }^{5}$. Previous studies have described the importance of this strength measurement for detecting pulmonary complications, morbidity and mortality in chronic cardiopulmonary disease patients; thus, it is an important tool in risk stratification ${ }^{6,7}$.

During RMT, intrathoracic pressure increases concomitant with linear load pressure ${ }^{4}$. A previous study demonstrated that overloading respiratory pressure produced beneficial hemodynamic and autonomic effects such as decreased sympathetic tone and improved arterial baroreceptor sensitivity ${ }^{8}$. Additionally, diaphragm length-tension curve alterations can modify vagal and sympathetic feedback on the sinus node via cardiovascular adjustment ${ }^{9}$. In this context, heart rate variability (HRV) has become an important method for assessing cardiovascular autonomic regulation ${ }^{10}$.

Moreover, Bainbridge, previously observed ${ }^{11}$ that dog heart rates (HRs) increased during ventricular filling, which corresponded to the inspiration phase. Thus, it is evident that respiration is a powerful modulator of HRV as well as baroreflex and chemoreflex sensitivity ${ }^{12}$.

It follows that breathing amplitude and muscle recruitment alterations may produce autonomic nervous system HR fluctuations. In parallel, Reis et al. ${ }^{13}$

\footnotetext{
${ }^{1}$ Laboratory of Cardiopulmonary Physical Therapy, Physical Therapy Department, Universidade Federal de São Carlos (UFSCar), São Carlos, SP, Brazil ${ }^{2}$ PneumoCardioVascular and Respiratory Muscle Performance Laboratory, Physical Therapy Department, Universidade Federal do Rio Grande do Norte (UFRN), Natal, RN, Brazil

Received: 11/06/2012 Revised: 12/14/2012 Accepted: 12/21/2012
} 
observed a significant association between respiratory muscle weakness and lower HRV indices in chronic obstructive pulmonary disease patients. RMT, can therefore improve autonomic HR modulation and is an important tool for reducing sympathetic activation, which is associated with higher rates of cardiovascular events and morbidity.

Because cardiovascular system modulation is noticeably affected by respiration ${ }^{12}$ in addition to the fact that RMT is an important therapeutic strategy and the aging process produces remarkable respiratory system alterations ${ }^{14-16}$, the present study investigated HR modulation during different inspiratory resistive loading intensities (mild, moderate and high) in healthy elderly men. The hypothesis of the present study was that lower inspiratory resistive loading intensities would promote marked and positive autonomic influences on the HR response, which would make it a powerful diagnostic and prognostic tool.

\section{Method}

\section{Study design and patient population}

This prospective, randomized, double-blind study was conducted with 33 healthy male subjects aged 60 to 75 years old. The study was approved by the Human Ethics Committee of Universidade Federal de São Carlos (UFSCar), São Carlos, SP, Brazil (109/2006) in compliance with the Declaration of Helsinki. Each volunteer was informed about the study and signed an informed consent form before participating.

The exclusion criteria were the following: body mass index $(\mathrm{BMI})>35 \mathrm{~kg} / \mathrm{m}^{2}$; systolic blood pressure (SBP) $>140 \mathrm{mmHg}$ or diastolic blood pressure (DBP) $>90 \mathrm{mmHg}$ (at rest); cardiac arrhythmias (atrial flutter or fibrillation, multiple ventricular or atrial ectopy, second or third degree atrioventricular block), smoking, medication use, left ventricular dysfunction, neurological or respiratory disorders and serious postural deviation in the chest such as severe scoliosis, kyphosis or hyperlordosis that could influence the respiratory pattern.

\section{Experimental procedures}

The study was performed at Cardiopulmonary Physical Therapy Laboratory and the Center for Physical Exercise Research at the UFSCar, Brazil. All of the procedures were performed between 8 a.m. and 12 p.m. with controlled temperature and relative humidity ( 22 to $24^{\circ} \mathrm{C}$ and 50 to $60 \%$, respectively).
The subjects were familiarized with the experimental environment and research personnel before the trials. Each volunteer was instructed to avoid caffeinated drinks on the day before and on the day of the test, to avoid physical exercise 24 hours prior to data collection, to eat a light meal on the morning of data collection and to sleep adequately (at least 8 hours) the night before the test.

\section{Clinical evaluation}

All of the participants underwent an evaluation consisting of an anamnesis involving clinical and family history and lifestyle habits, a physical evaluation to evaluate posture, vital sign measurements (respiratory rate, heart rate and systolic and diastolic blood pressure), anthropometric measurements (weight and height), a conventional resting electrocardiogram (FUNBEC, São Paulo, SP, Brazil), laboratory exams (blood glucose, cholesterol, triglyceride, uric acid, and creatine levels as well as urinalysis) and a cardiologist-administered maximal or symptom-limited exercise test to evaluate cardiovascular response integrity.

\section{Respiratory muscle strength and inspiratory resistive loading protocol}

Maximal inspiratory pressure (MIP) was obtained by measuring the difference in the residual volume from the total lung capacity; maximal expiratory pressure (MEP) was obtained by measuring the difference in the total lung capacity from the residual volume. During evaluation, the subjects sat and wore nose clips as well as a mouthpiece that was connected to a manual shutter apparatus with maximal pressure as measured by an aneroid-gauge manometer $\left( \pm 300 \mathrm{cmH}_{2} \mathrm{O}\right)$ (GER-AR, São Paulo, SP, Brazil). The volunteers were asked to perform MIP and MEP efforts against an obstructed mouthpiece with a small leak to prevent them from closing their glottis during the maneuver. Patients sustained their maximal effort for one second, and the best of three consecutive attempts was used to determine MIP. The percent-predicted values were derived from this measurement ${ }^{17}$.

After determining the maximal respiratory pressures, the inspiratory effort protocol was applied, which featured three inspiratory resistive loading intensities: 30, 60 and 80\% MIP. During the protocol, the volunteer sat in a chair, wore a nose clip and made inspiratory efforts using a previously adjusted inspiratory resistance device (POWERbreathe, Southam, UK). The load order was applied randomly 
by lots. The volunteers and examiner were blinded to the applied loads.

The maneuver was performed during the appropriate breathing time (inspiratory and expiratory times). To accomplish this, the volunteers were previously instructed to begin on a verbal command, which corresponded to the appropriate respiratory cycle phase. The volunteer was verbally encouraged to inhale for 2 seconds and exhale for 3 seconds, similar to a physiological breathing pattern, completing 12 breaths per minute. A wall clock with a second hand was used to maintain the cycle synchrony and respiratory rate. Moreover, the researcher provided verbal feedback based on the ECG signal and HR plot on the computer monitor, which confirmed whether the respiratory cycle had been performed correctly. Each effort level was performed for 4 minutes and was separated by 5 minutes of rest and 5 minutes of recovery.

\section{Heart rate and $R-R$ interval data acquisition}

HR and R-R interval (R-Ri) data were recorded using a Polar S810i heart rate monitor (Polar Electro TM, Kempele, Finland) with a $1000 \mathrm{~Hz}$ sampling frequency ${ }^{18}$ at rest and during the exercise protocol. Subjects were asked to not participate in physical exercise or ingest caffeinated products $24 \mathrm{~h}$ prior to the experiment. Initially, the subjects rested in a sitting position for $10 \mathrm{~min}$. Measurements were then obtained as follows: 1) Rest: Seated resting spontaneous breathing (SB) measurements were obtained for 5 minutes before any exercise was performed; and 2) Exercise: Exercise measurements were obtained for 4 minutes at each intensity (30, 60 and $80 \%$ of MIP).

\section{Signal processing and HRV analysis}

After acquisition, the signals were transferred to the Polar Precision Performance Software, visually inspected and corrected for ectopic beats (i.e., premature, supraventricular and ventricular). Periods with more than $10 \%$ correction were excluded. Time series data were processed using Kubios HRV Analysis software (MATLAB, version 2 beta, Kuopio, Finland). For analysis, a HR and R-Ri section at each exercise intensity that included 6 respiratory cycles was selected by visual inspection according to the European Society of Cardiology and the North American Society of Pacing and Electrophysiology Task Force of criteria ${ }^{10}$. A 5 min section SB in pre-exercise rest was also analyzed.

The HRV was analyzed using mathematical and statistical models within the time and frequency domains and with nonlinear models. Time domain analysis included: mean R-Ri, mean R-Ri for normal beats; the NN interval standard deviation (SDNN), the normal R-Ri standard deviation, and the root mean square of the squares of the differences between successive R-Ri (rMSSD) in ms, which was representative of parasympathetic activity. Frequency domain analysis included low frequency (LF) and high frequency (HF) bands in absolute and normalized units. The LF $(0.04$ to $0.15 \mathrm{~Hz})$ has been associated with predominant sympathetic modulation while the HF ( 0.15 to 0.4 ) has been associated with parasympathetic modulation ${ }^{10}$.

For nonlinear HRV analysis ${ }^{19}$, we used the Poincaré plot indices SD1 and SD2 (the Poincaré plot perpendicular standard derivation and along the line of identity, respectively), which are representative of parasympathetic autonomic modulation and total HRV, respectively.

\section{Statistical analysis}

After performing the Shapiro-Wilk test, normally distributed variables were expressed as the mean values plus or minus standard deviation or standard error as required. Repeated-measures analysis of variance (ANOVA) was used to compare the HRV indices among $30 \%, 60 \%$ and $80 \%$ of MIP and rest. When the difference was significant, a post-hoc Tukey-Kramer test was used. The probability of a type I error was established at 5\% for each test $(\mathrm{p}<0.05)$. The data were analyzed using Statistica for Windows (Stat Soft Inc., 2000) and Instat (GraphPad Software, 2000).

\section{Results}

Of the 34 subjects referred for evaluation, only 25 were included in the study. Of those excluded, 4 refused to participate for personal reasons, 2 presented with systolic blood hypertension $>140 \mathrm{~mm}$ $\mathrm{Hg}$ and 3 had coronary artery disease as diagnosed by catheterization.

The age and anthropometric data (i.e., body weight, height, BMI) as well as the clinical data such as resting HR, blood pressure, MIP, the expected maximal inspiratory pressure (MIPexp), the MIP/ MIPexp ratio (MIP\%), MEP, the expected maximal expiratory pressure (MEPexp) and the MEP/MEPexp ratio (MEP\%) are presented in Table 1. The MIP and MEP values in this study were not significantly different $(p>0.05)$ from those of the Brazilian population (Table 1). 
Table 1. Demographic, anthropometric and clinical data.

\begin{tabular}{lc}
\hline & $\mathbf{n = 2 5}$ \\
Age (years) & $66 \pm 4$ \\
Body mass $(\mathrm{kg})$ & $72 \pm 9$ \\
Height $(\mathrm{cm})$ & $167 \pm 5$ \\
BMI $\left(\mathrm{kg} / \mathrm{m}^{2}\right)$ & $25.3 \pm 2$ \\
Clinical data & \\
HR $(\mathrm{bpm})$ & $71 \pm 4$ \\
RR $(\mathrm{cpm})$ & $14 \pm 2$ \\
SBP $(\mathrm{mmHg})$ & $123 \pm 12$ \\
DBP $\left(\mathrm{mmHg}^{2}\right)$ & $76 \pm 7$ \\
MIP $\left(\mathrm{cmH}_{2} \mathrm{O}\right)$ & $97 \pm 30$ \\
MEP $\left(\mathrm{cmH}_{2} \mathrm{O}\right)$ & $124 \pm 25$ \\
MIP expected $\left(\mathrm{cmH}_{2} \mathrm{O}\right)$ & $105 \pm 5$ \\
MEP expected $\left(\mathrm{cmH}_{2} \mathrm{O}\right)$ & $112 \pm 3$ \\
MIP \% & $92 \pm 38$ \\
MEP \% & $96 \pm 46$ \\
\hline
\end{tabular}

Values are expressed as the mean $\pm \mathrm{SD}$. BMI=body mass index; $\mathrm{HR}=$ heart rate; $\mathrm{RR}=$ respiratory rate; $\mathrm{SBP}=$ systolic blood pressure; $\mathrm{DBP}=$ diastolic blood pressure; $\mathrm{MIP}=$ maximum inspiration pressure; $\mathrm{MEP}=$ maximum expiration pressure; $\mathrm{MIP} \%=$ ratio $\mathrm{MIP} / \mathrm{MIP}$ expected; $\mathrm{MEP} \%=$ ratio MEP/MEPexpected.
Table 2 demonstrates the HRV indices that were obtained during the three different inspiratory loads that have been divided into HRV analysis in the time domain (TD), frequency domain (FD) and nonlinear analysis. The HRV, rMSSD, SD1 and HF parasympathetic indices demonstrated lower values during $80 \%$ inspiratory resistive loading than at $30 \%$ of MIP (Table 2).

Figure 1 demonstrates the rMSSD, LF and HF during SB compared with 30,60 and $80 \%$ of MIP in the controlled respiratory breathing protocol. We observed significantly lower values for rMSSD and HF in SB than at 30, 60 and $80 \%$ of MIP. However, rMSSD at $30 \%$ of MIP was higher than at 60 and $80 \%$. In addition, LF was higher during SB at 30, 60 or $80 \%$ of MIP.

Figure 2 demonstrates the Poincaré plot during the three study conditions, which revealed lower chaotic behavior for autonomic cardiac sinus node modulation at $30 \%$ of MIP. Significant differences ( $p>0.05)$ in representative sympathetic heart rate indices were not found between the three imposed loads.

Table 2. Heart rate variability at different inspiratory load levels during controlled respiratory breathing.

$\begin{array}{llll}30 \% & 60 \% & 80 \% & \text { P value }\end{array}$

\section{Time domain HRV}

\begin{tabular}{|c|c|c|c|c|}
\hline Mean RR (ms) & $781 \pm 23$ & $789 \pm 19$ & $768 \pm 24$ & 0.24 \\
\hline Mean HR (1/min) & $79 \pm 3$ & $77 \pm 2$ & $80 \pm 3$ & 0.15 \\
\hline rMSSD (ms) & $25 \pm 3$ & $22 \pm 2$ & $19 \pm 2 *$ & 0.02 \\
\hline SDNN (ms) & $39 \pm 3$ & $36 \pm 4$ & $34 \pm 3$ & 0.40 \\
\hline
\end{tabular}

\section{Frequency domain HRV}

$\begin{array}{lcccc}\mathrm{LF}\left(\mathrm{ms}^{2}\right) & 146 \pm 36 & 302 \pm 130 & 197 \pm 61 & 0.40 \\ \mathrm{HF}\left(\mathrm{ms}^{2}\right) & 447 \pm 99 & 337 \pm 67 & 228 \pm 61 * & 0.004 \\ \mathrm{LF} / \mathrm{HF} & 1.2 \pm 0.4 & 1.2 \pm 0.3 & 1.8 \pm 0.7 & 0.07\end{array}$

\section{Nonlinear HRV}

$\begin{array}{lcccr}\text { SD1 } & 18 \pm 2.3 & 16 \pm 1.6 & 13 \pm 1.7 * & 0.02 \\ \text { SD2 } & 53 \pm 4.4 & 49 \pm 5 & 46 \pm 4 & 0.35 \\ \text { ApEn } & 0.91 \pm 0.02 & 0.97 \pm 0.01 & 0.93 \pm 0.02 & 0.08\end{array}$

Values are expressed as the mean \pm SE. Mean RR=mean distance between the RR intervals; Mean HR=mean heart rate; $\mathrm{rMSSD}=\mathrm{square}$ root of the mean of the sum of the squares of the differences between adjacent RR intervals; SDNN=standard deviation of all RR intervals; LF=low frequency spectral component; $\mathrm{HF}=$ high frequency spectral component; $\mathrm{LF} / \mathrm{HF}=$ low-high frequency power ratio; $\mathrm{SD} 1=$ related to the fast beatto-beat variability; SD2=describes the longer-term RR interval variability; ApEn=quantifies system entropy, i.e., refers to HR irregularity and predictability. *Significant difference compared to $30 \%$. 

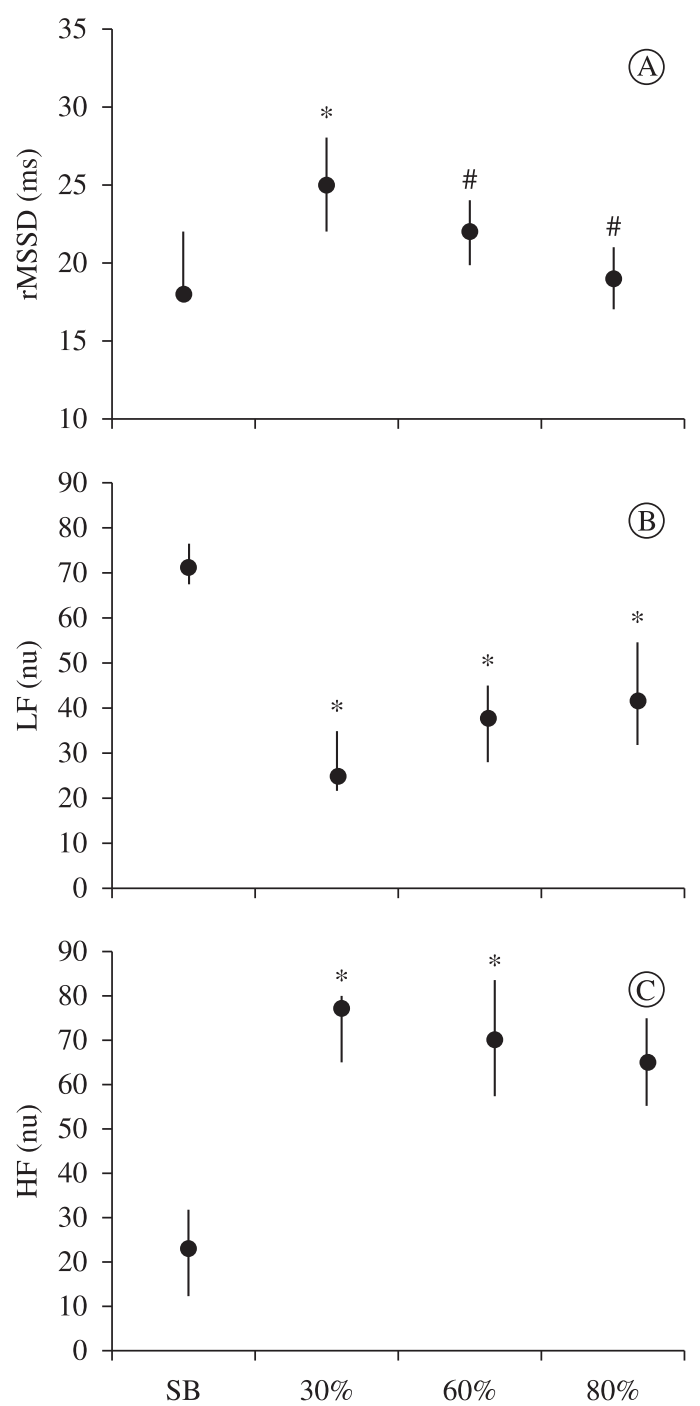

Figure 1. HRV indices in (A) rMSSD, (B) LF - low frequency and (C) HF - high frequency compared with spontaneous breathing (SB) and different maximal inspiratory load percentages. $* \mathrm{p}<0.05$ compared with SB; \# p $<0.05$ compared with 60 and $80 \%$.

\section{Discussion}

The purpose of this study was to investigate acute HR modulation during different inspiratory resistive loading intensities (mild, moderate and high) in healthy elderly men. The main findings of this study demonstrated that load increment produced significantly lower values for representative parasympathetic cardiac modulation indices in the healthy elderly. Significantly reduced rMSSD, HF, and SD1 values were determined at $80 \%$ of MIP compared with $30 \%$ of MIP during inspiratory resistive loading. These findings suggest that lower inspiratory resistive loading intensities promote marked, positive improvement in parasympathetic sinus node modulation.
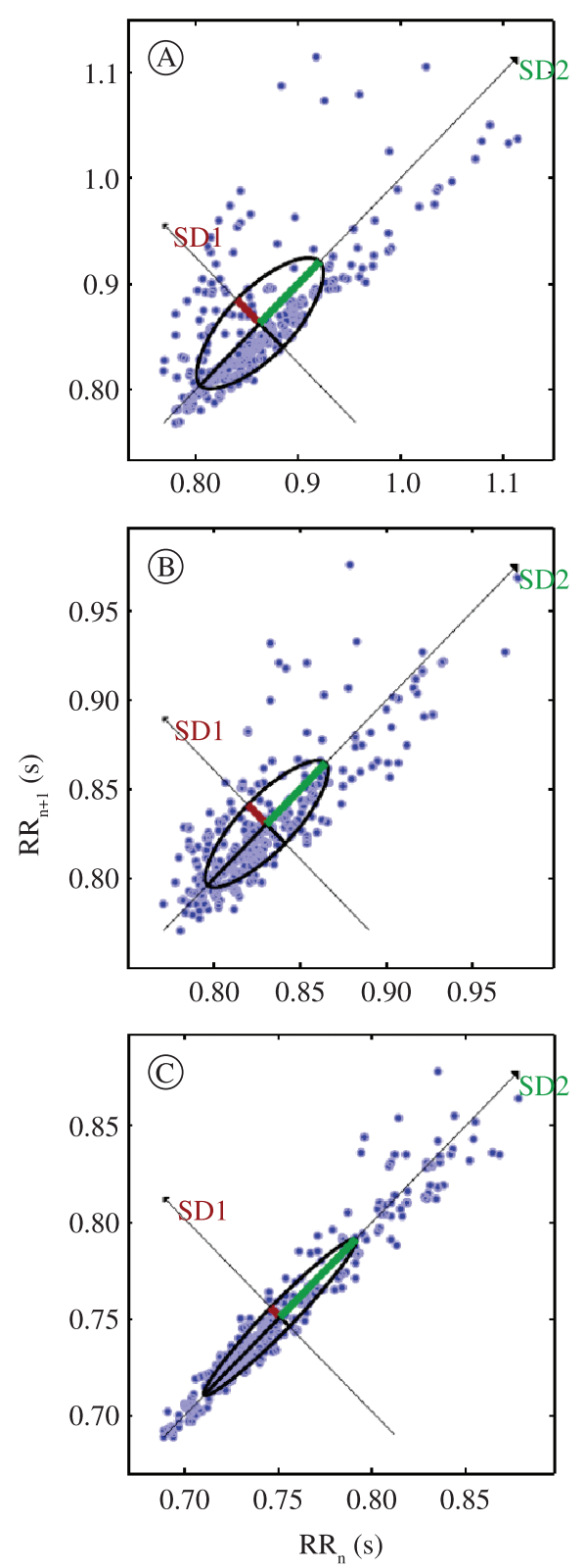

Figure 2. Poincaré-plot in different inspiratory exercise loads: (A) $30 \%$, (B) $60 \%$ and (C) 80\%. SD1: standard deviation of the instantaneous beat-to-beat R-R interval variability. SD2: standard deviation of the long term R-R interval variability.

Although inspiratory muscle training is the most widely used method, cardiovascular adjustment during different inspiration intensities remains unstudied. In a previous study on cardiovascular adjustment during respiratory resistive loads in healthy subjects ${ }^{20}$, the authors analyzed HRV during four different resistive loads that were applied throughout the entire breathing cycle. In seven healthy subjects aged 19 to 55 , resistive respiratory loads of 3.25 to $12.5 \mathrm{cmH}_{2} \mathrm{O}$ were applied during an average 
of 50 breaths and the HRV was analyzed. These authors observed a progressive increase in both LF and HF spectral components. Furthermore, the LF/ HF demonstrated a progressive load intensity increase that exceeded a sympathovagal balance of 1 at 8.25 and $12.5 \mathrm{cmH}_{2} \mathrm{O}$. These results by Calabrese et al. ${ }^{20}$ can only be partially compared with ours.

McConnell and Griffiths ${ }^{21}$ also evaluated the influence of different MIP percentages (50, 60, 70, 80 and $90 \%$ ) in eight healthy males and observed that beginning at $60 \%$ MIP, HR and blood pressure increased. In addition, Sheel et al. ${ }^{22}$ demonstrated that HR increased during an MIP protocol, which was time-dependent. These authors ${ }^{23}$ also observed that the diaphragm metaboreflex is intensively activated during loaded breathing until task failure, which induces sympathetic activation and peripheral vasoconstriction that may limit exercise performance.

In this context, important cardiovascular behavior alterations have been attributed to local metabolic changes during loaded breathing. Afferent receptors, mechanical deformation, temperature elevation and vascular distension during muscular contractions may be stimulated by metabolic products ${ }^{24}$. Respiratory muscle contraction causes changes in local metabolic homeostasis; consequently, myelinated group III and unmyelinated IV afferent nerve fibers trigger a cardiovascular adjustment with increased sympathetic activity to accompany the metabolic demand $d^{25}$. However, during an endurance protocol, autonomic responses could indicate the best intensity at which to train inspiratory muscles because of improved vagal activation. We demonstrated that lower inspiratory resistive loading intensities promote parasympathetic modulation compared with higher intensities. In agreement with these results, Callegaro et al. ${ }^{26}$ observed that RMT at $60 \%$ of MIP significantly attenuated the inspiratory muscle metaboreflex.

One experimental study ${ }^{8}$ involving rats with heart failure demonstrated improved cardiovascular function after 6 weeks of RMT as assessed by decreased left ventricular end-diastolic pressure, sympathetic tone, right ventricular hypertrophy and lung and hepatic congestion as well as increased vagal effects and arterial baroreceptor sensitivity.

Nobre et al. ${ }^{27}$ observed that EMG (electromyography) activity in lower rib cage muscles increased during progressive respiratory workloads. This may be directly related to high respiratory muscle recruitment because at intensities closer to the MIP, the diaphragm and accessory muscles (scalene muscles, sternocleidomastoid, pectoralis minor, external intercostals) increased intrathoracic pressure and induced intense sympathetic activation.

Another plausible explanation for these results is that because this intrathoracic pressure increase is generated at high intensities during each breathing cycle, it may reduce venous return to the right side of heart and act directly on the sinus node to reduce right atrial wall stretch ${ }^{28}$. Blood damming in the pulmonary vascular bed that occurred because of the sudden intrathoracic pressure increase may also have reduced the blood volume that was sent to the left ventricle with a consequent instantaneous blood pressure drop, leading to baroreflex vagal withdrawal ${ }^{29,30}$. Another explanation could be that the reduced vagal modulation during the higher inspiratory resistive loading in this protocol was induced by the intense effort that was required to sustain $80 \%$ of MIP for the pre-determined time. These results can be compared with those occurring in HRV in inspiratory maneuvers at high volumes such as the respiratory sinus arrhythmia accentuation maneuver (RSA-M).

In contrast with the RSA-M (in which the inspired volume increased slowly over 5 seconds), the inspired volume increased rapidly at $80 \%$ of MIP because the inspiratory time was $2 \mathrm{~s}$. Additionally, in the present study, the R-Ri was also collected differently than the RSA-M (which is standardized at 6 breaths per minute). Thus, even at higher exercise intensities and inspiratory volumes, the lung inflation-mediated vagal response was reduced in relation to higher loads.

Finally, our results demonstrated that lower inspiratory resistive loading intensities provided greater safety for the study population because it promoted a greater vagal response, which indicates a powerful cardioprotective effect. Furthermore, we emphasize the importance of our results for the elderly population because the RMS is decreased in these individuals. Thus, although our sample did not present respiratory muscle weakness compared to the reference values, we believe that future studies involving this population with inspiratory muscle weakness will be worthwhile for greater understanding of the autonomic HR responses.

Some study limitations should be considered. First, control of tidal volume measurement during the inspiratory control, which was not performed in this study, could have contributed to result consolidation and interpretation. Second, baroreflex and/or chemoreflex were not evaluated, which could also have contributed to the interpretation of our results. Finally, the R-Ri was not controlled during the resting HRV assessment, which comparing these data with the proposed protocol difficult. 
In conclusion, our results suggest that lower inspiratory efforts produce higher HRV. These findings represent important clinical applications because low RMT intensities can produce greater parasympathetic HR modulation in this population. Thus, we should choose the most appropriate load for achieving the most beneficial autonomic effects, which are associated with reduced cardiovascular event and morbidity incidence.

However, future studies are needed to confirm whether the RMT can enhance vagal modulation at low inspiratory muscle training intensity. Moreover, other studies are needed to confirm these findings in other populations such as in chronic obstructive pulmonary disease and cardiac heart failure, where respiratory muscle weakness is commonly present.

\section{- Acknowledgements}

We acknowledge the UFSCar Cardiopulmonary Laboratory staff for their enthusiastic help. Special thanks are given to the subjects for their participation. Support for this work was provided by grants from Conselho Nacional de Desenvolvimento Científico e Tecnológico (CNPq) and Fundação de Amparo a Pesquisa do Estado de São Paulo (FAPESP) $n^{\circ} 2009 / 01842-0$.

\section{References}

1. Westerdahl E, Lindmark B, Almgren SO, Tenling A. Chest physiotherapy after coronary artery bypass graft surgery: a comparison of three different deep breathing techniques. J Rehabil Med. 2001;33(2):79-84. http:// dx.doi.org/10.1080/165019701750098920

2. Westerdahl E, Lindmark B, Eriksson T, Hedenstierna $\mathrm{G}$, Tenling, A. The immediate effects of deep breathing exercises on atelectasis and oxygenation after cardiac surgery. Scand Cardiovasc J. 2003;37(6):363-7. http:// dx.doi.org/10.1080/14017430310014984

3. Stein R, Maia CP, Silveira AD, Chiappa GR, Myers J, Ribeiro JP. Inspiratory muscle strength as a determinant of functional capacity early after artery bypass graft surgery. Arch Phy Med Rehabil. 2009;90(10):1685-91. http:// dx.doi.org/10.1016/j.apmr.2009.05.010

4. Dall'ago P, Chiappa GR, Güths H, Stein R, Ribeiro JP. Inspiratory muscle training in patients with heart failure and inspiratory muscle weakness: a randomized trial. J Am Coll Cardiol. 2006;47(4):757-63. http://dx.doi. org/10.1016/j.jacc.2005.09.052

5. Neder JA, Andreoni S, Lelario MC, Nery LE. References values for lung function tests. II. Maximal respiratory pressures and voluntary ventilation. Braz J Med Biol Res. 1999;32(6):719-27. http://dx.doi.org/10.1590/ S0100-879X1999000600007
6. Meyer FJ, Borst MM, Zugck C, Kirsche A, Schellberg $\mathrm{D}$, Kubler, et al. Respiratory muscle dysfunction in congestive heart falure: clinical correlation and prognostic significante. Circulation. 2001;103(17):2153-8. http:// dx.doi.org/10.1161/01.CIR.103.17.2153

7. Ribeiro JP, Chiappa GR, Neder JA, Frankestein L. Respiratory muscle function and exercise intolerance in heart failure. Curr Heart Failure Rep. 2009;6(2):95-101. http://dx.doi.org/10.1007/s11897-009-0015-7

8. Jaenisch RB, Hentschke VS, Quagliotto E, Cavinato PR, Schmeing LA, Xavier LL, et al. Respiratory muscle training improves hemodynamics, autonomic function, baroreceptor sensitivity, and respiratory mechanics in rats with heart failure. J Appl Physiol. 2011;111(6):1664-70. http://dx.doi.org/10.1152/japplphysiol.01245.2010

9. Grossman P, Wilhelm FH, Spoerle M. Respiratory sinus arrhythmia, cardiac vagal control and daily activity. Am J Physiol. 2004;287:H728-H34.

10. European Society of Cardiology, North American Society of Pacing Electrophysiology. Task force. Heart rate variability. Standards of measurement, physiological interpretation, and clinical use. Circulation. 1996;93:104365. http://dx.doi.org/10.1161/01.CIR.93.5.1043

11. Bainbridge FA. The influence of venous filling upon the rate of the heart. J Physiol. 1915;50:65-84.

12. Bernardi L, Porta C, Gabutti A, Spicuzza L, Sleight P. Modulatory effects of respiration. Auton Neurosci. 2001;90(1-2):47-56. http://dx.doi.org/10.1016/ S1566-0702(01)00267-3

13. Reis MS, Arena R, Deus AP, Simões RP, Catai AM, Borghi-Silva A. Deep Breathing and respiratory muscle weakness in COPD. Clinics. 2010;65(4):369-75. http:// dx.doi.org/10.1590/S1807-59322010000400004

14. Simões RP, Deus APL, Auad MA, Dionisio J, Mazzonetto M, Borghi-Silva A. Maximal respiratory pressure in healthy 20 to 89 year-old sedentary individuals of central São Paulo State. Rev Bras Fisioter. 2010;14(1):60-7. http:// dx.doi.org/10.1590/S1413-35552010000100010

15. Parreira VF, Bueno CJ, França DC, Vieira DS, Pereira DR, Britto RR. Breathing pattern and thoracoabdominal motion in healthy individuals: influence of age and sex. Rev Bras Fisioter. 2010;14(5):411-6. http://dx.doi.org/10.1590/ S1413-35552010000500010

16. Simões LA, Dias JMD, Marinho KC, Pinto CLLR, Britto RR. Relationship between functional capacity assessed by walking test and respiratory and lower limb muscle function in community-dwelling elders. Rev Bras Fisioter. 2010;14(1):24-30. http://dx.doi.org/10.1590/ S1413-35552010000100005

17. American Thoracic Society, European Respiratory Society. ATS/ERS Statement of Respiratory Muscle Testing Guidelines. Am J Respir Crit Care Med. 2002;166(4):518624. http://dx.doi.org/10.1164/rccm.166.4.518

18. Nunan D, Donovan G, Jakovljevic DG, Hodges LD, Sandercock GR, Brodie DA. Validity and reliability of short-term heart-rate variability from the Polar S810. Med Sci Sports Exerc. 2009;41(1):243-50. http://dx.doi. org/10.1249/MSS.0b013e318184a4b1 
19. Stein PK, Reddy A. Non-linear heart rate variability and risk stratification in cardiovascular disease. Indian Pacing Electrophysiol J. 2005;5(3):210-20.

20. Calabrese P, Perrault H, Dinh TP, Eberhard A, Benchetrit G. Cardiorespiratory interactions during resistive load breathing. Am J Physiol Regul Integr Comp Physiol. 2000;279(6):R2208-13.

21. McConnell AK, Griffiths LA. Acute cardiorespiratory responses to inspiratory pressure threshold loading. Med Sci Sports Exerc. 2010;42(9):1696-703. http://dx.doi. org/10.1249/MSS.0b013e3181d435cf

22. Sheel AW, Derchak PA, Morgan BJ, Pegelow DF, Jacques AJ, Dempsey JA. Fatiguing inspiratory muscle work causes reflex reduction in resting leg blood flow in humans. J Physiol 2001;537(Pt 1):277-89. http://dx.doi. org/10.1111/j.1469-7793.2001.0277k.X

23. Sheel AW, Derchak PA, Pegelow DF, Dempsey JA. Threshold effects of respiratory muscle work on limb vascular resistance. Am J Physiol Heart Circ Physiol. 2002;282(5):H1732-8.

24. Haouzi P, Chenuel B, Huszczuk A. Sensing vascular distension in skeletal muscle by slow conducting afferent fibers: neurophysiological basis and implication for respiratory control. J Appl Physiol. 2004:96:407-18. http:// dx.doi.org/10.1152/japplphysiol.00597.2003

25. Roseguini BT, Alves CN, Chiappa GR, Stein R, Knorst MM, Ribeiro JP. Attenuation of muscle metaborreflex in chronic obstrutive pulmonary disease. Med Sci Sports Exerc. 2008;40(1):9-14.
26. Callegaro CC, Ribeiro JP, Tan CO, Taylor JA. Attenuated inspiratory muscle metaboreflex in endurance-trained individuals. Respir Physiol Neurobiol. 2011;177(1):24-9. http://dx.doi.org/10.1016/j.resp.2011.03.001

27. Nobre ME, Lopes F, Cordeiro L, Marinho PE, Silva TN, Amorim $\mathrm{C}$, et al. Inspiratory muscle endurance testing: pulmonary ventilation and electromyographic analysis. Respir Physiol Neurobiol. 2007;155(1):41-8. http://dx.doi. org/10.1016/j.resp.2006.04.005

28. Tulppo MP, Makikallio TH, Takala TE, Seppanen T, Huikuri HV. Quantitative beat-to-beat analysis of heart rate dynamics during exercise. Am J Physiol Heart Circ Physiol. 1996;271:H244-52.

29. Tulppo MP, Makikallio TH, Takala TE, Seppanen T, Huikuri HV. Vagal modulation of heart rate during exercise: effects of age and physical fitness. Am J Physiol. 1998;274(2):H424-9.

30. Hirsch JA, Bishop B. Respiratory sinus arrhythmia in humans: how breathing patterns modulates heart rate. Am J Physiol. 1981;10:620-9.

\section{Correspondence}

Audrey Borghi-Silva

Universidade Federal de São Carlos

Departamento de Fisioterapia

Rodovia Washington Luis, Km 235, Monjolinho

CEP 13565-905, São Carlos, SP, Brazil

e-mail: audrey@ufscar.br 